\title{
Bibliographie
}

Courtes, G. (1960).-Ann. d'Astr. 23: 115-217.

Courtes, G. (1962a).-C.R. Acad. Sci. (Paris) 254: 1738-40.

Countes, G. (1962b).-Astronomie 76: 224-31.

Courtes, G. (1962c).-Astronomie 76: 250-8.

Courtes, G. $(1963 a)$.- - Paper presented to Symposium on Instrumental Astronomy at the 113th Meeting of the American Astronomical Society, Tucson, Arizona.

Courtes, G. (1963b).-Astronomie 77 : (in press).

Courtes, G., et Cruverlier, P. (1960a)._- "Les recherches galactiques et extragalactiques et la photographie electronique." = Colloque Int. CNRS no. 95. [Paris 1959.] Ann. d'Astr. 23: 419-21.

Courtss, G., et Cruvellier, P. (1960b).-C.R. Acad. Sci. (Paris) 251 : 2470-2.

Rodgers, A. W., Campbelu, C. T., Whiteoak, J. B., Bailey, H. H., and Hunt, V. O. (1960)."An Atlas of H-alpha Emission in the Southern Milky Way." (Mount Stromlo Observatory: Canberra.)

\section{ON THE HII REGIONS IN GALAXIES}

\author{
J. L. SERSIC* \\ Observatorio Astronomico, Córdoba
}

In the present paper we shall consider that the HII complexes observed in galaxies may be interpreted as the resulting Strömgren volume produced by a cluster or association of exciting stars. If $\rho_{\mathbf{H}}$ is the density of the hydrogen surrounding the cluster or association and $I$ the number of ionizing quanta $(\lambda<912 \AA)$ emitted by the stars, the ionized volume becomes

$$
V_{i} \propto I / \rho_{\mathrm{H}}^{2} \text {. }
$$

It is possible to prove that the number of ionizing quanta $I(L)$ emitted by a star is proportional to the bolometric luminosity $L$ for spectral types earlier than B3, so that, if $\phi(L)$ is the actual luminosity function of the cluster (in stars per cubic parsec) and $V$ a representative volume, then

$$
I \propto V \int_{L_{1}}^{L} \phi(L) L \mathrm{~d} L .
$$

Let us now assume that the present luminosity function results from a universal luminosity function $\psi(L)$ valid for all the galaxies, then the preceding expression becomes

$$
I \propto V \int_{L_{1}}^{L} \mathrm{~d} L \int_{t-\tau(L)}^{t}(\mathrm{~d} N / \mathrm{d} T) \psi(L) L \mathrm{~d} t,
$$

where $L_{1}$ is the luminosity of the B3 stars. Here $\tau(L)$ is the lifetime of the stars with luminosity $L$ in the main sequence. By assuming now a constant creation rate $(\mathrm{d} N / \mathrm{d} t)$ in a short interval such as $\tau(L) \propto M / L$, we have

$$
I \propto V(\mathrm{~d} N / \mathrm{d} T) \int_{L_{1}}^{L} \psi(L) M \mathrm{~d} L,
$$

* Presented by A. W. Rodgers. 
an expression for $I$ that, carried to (1), results in

$$
\xi \propto \frac{1}{\rho_{\mathrm{H}}^{2}} \frac{\mathrm{d} N}{\mathrm{~d} t} \int_{L_{1}}^{L} \psi(L) M(L) \mathrm{d} L .
$$

The meaning of $\xi$ is the fraction of space that is ionized by exciting stars. If we disregard the lack of homology of the main sequence stars, an alternative form for $\xi$ is obtained observing that

$$
N \int_{L_{1}}^{L} \dot{\psi}(L) M(L) \mathrm{d} L
$$

is interpreted as the mass density $\rho_{i}$ of the ionizing stars, so that

$$
\xi \propto \frac{1}{\rho_{\mathrm{H}}^{2}} \cdot \frac{\mathrm{d} \rho_{i}}{\mathrm{~d} t}
$$

establishes a relation between the ionized fraction of space and the rate of formation of exciting stars.

The foregoing considerations point to the HII regions in galaxies as indicators of the rate of formation of massive-exciting-stars. This fact is well understood when we notice that $\mathrm{d} \rho_{i} / \mathrm{d} t$ must surpass a certain definite value in a certain region of the space in order to have $\xi \geqslant 1$, that is to say, be in a fully ionized region. The spatial distribution, frequency of diameters, and total number of HII regions in galaxies are indicators of the present rate of formation of massive stars. Observational material is still needed although we shall give some hints suggested by actual observations.

Assume as usual that the rate of stellar formation is related to the gas density by

$$
\frac{\mathrm{d} N}{\mathrm{~d} t}=K \rho_{\mathrm{H}}^{n}
$$

where $N$ is the total number of stars formed in a given cluster or association, not necessarily in a whole galaxy. The coefficient $K$ is supposed to be independent of time. The fraction of space ionized by a cluster or association is then

$$
\xi \propto \rho \mathrm{H}^{\mathrm{n}-2} \int_{L_{1}}^{L} \psi(L) M(L) \mathrm{d} L,
$$

and we shall now consider different values of $n$. The case $n<2$ should be discarded because it is difficult to imagine large HII regions in the outer, low hydrogen density fringes of the galaxies. Supposing now $n=2$, the ionized volume would be independent of the gas density and

$$
G(L)=\int_{L_{1}}^{L} \psi(L) M(L) \mathrm{d} L
$$

will be the determining parameter for $\xi$. With $n>2, \xi$ depends on the gas density and would also depend on $G(L)$. In order to decide between the two cases, let us consider the distribution of ionized hydrogen in the Galaxy as derived by radio-astronomical techniques (Westerhout 1958). Figure 1 shows the fraction of the space that is ionized in the galactic disk in correspondence to the distribution of the total gas density. The ionized fraction of space follows a trend qualitatively close to the total density distribution. We do not see any immediate reason for such a dependence between $G(L)$ and the gas density and we think that it is simpler to assume $n>2$. 
A value $n=3$ has been suggested by Schmidt (1962) for the rate of stellar formation of massive stars with more than 7 solar masses, in order to conform to the luminosity function in young clusters.

Let us now apply the preceding concepts to a galaxy as a whole. If the volume $V$ of the galaxy is taken as a unit, the fraction of space ionized by the clusters or associations with an upper luminosity limit $L$ in that galaxy is

$$
\xi \propto K M_{\mathrm{H}}^{n-2} G(L)
$$

and the corresponding ionized volume becomes

$$
V_{i} \propto K . V . G(L) . M_{\mathrm{H}}^{n-2},
$$

a relation connecting the volume $V_{i}$ ionized by the brightest star clusters and associations with the total hydrogen mass of a galaxy.

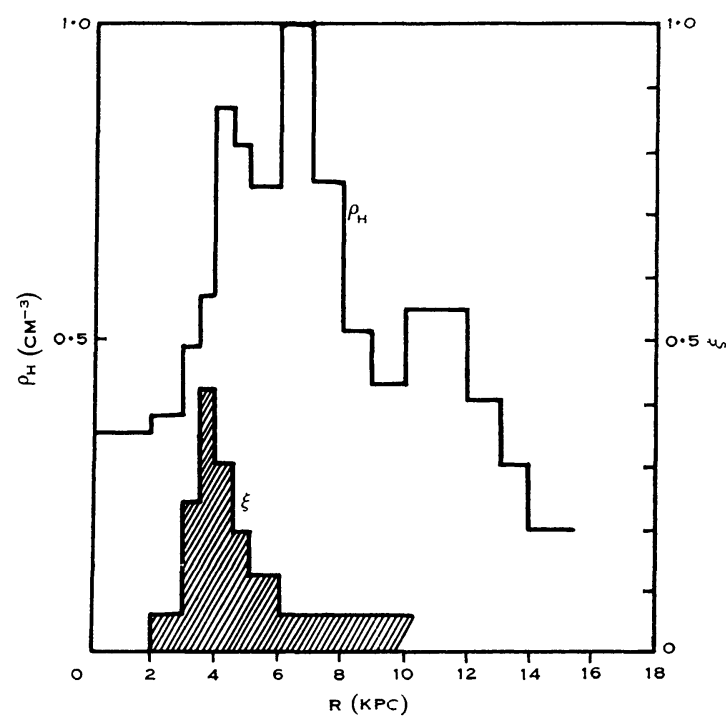

Fig. 1

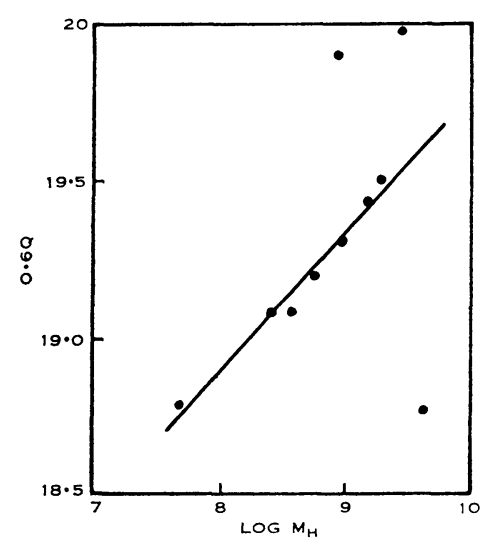

Fig. 2

Fig. 1.-Distribution of $\mathrm{HI}$ and $\mathrm{HII}$ with distance from galactic centre.

Fig. 2.-Relation between diameter of three largest HII regions and total mass of HI.

Let us now consider the function $G(L)$. Table 1 has been calculated with Sandage's original luminosity function $\psi(L)$ and masses (Sandage 1957) and choosing $L_{1}$ as the luminosity corresponding to B3 stars $\left(M_{V}=-3\right)$. Table 1 shows that $G(L)$ becomes rather insensitive to changes in the argument when $L$ is adequately large. That is precisely the case for the largest HII regions in a galaxy, so $G(L)$ would be only proportional to the number of HII regions per unit volume in a galaxy. Then $V . G(L)$ is proportional to the total number of the largest HII regions in the galaxy considered. The average volume of the largest HII regions would, then, be

$$
V_{i} \propto M_{\mathbf{H}}^{n-2} \text {. }
$$

In another place (Sérsic 1960) we have measured the diameters of the three largest HII regions in several galaxies, the quantity $Q=5 \log D+21 \cdot 55$ (where $D$ 
is the mean diameter of the three largest ones in parsec) and the hydrogen mass $M_{\mathrm{H}}$ is given in Table 2 where it has been estimated for galaxies. Figure 2 shows that there exists a linear relationship between $0.6 Q$ and $\log M_{\mathrm{H}}$,

$$
0 \cdot 6 Q=0 \cdot 55 \log M_{\mathrm{H}}+14 \cdot 5,
$$

for galaxies of a later than $\mathrm{Sb}^{-}$type. The slope of the relation suggests a value of $n$ close to $2 \cdot 5$. The galaxies of type earlier than $\mathrm{Sb}^{+}$appear to fall below the

Table 1

VALUES OF $G(L)$

\begin{tabular}{c|c|c|c}
$M_{V}$ & $\log G(L)+$ const. & \multicolumn{1}{|c|}{$M_{V}$} & $\log G(L)+$ const. \\
\hline$-3 \cdot 0$ & - & $-3 \cdot 5$ & $-0 \cdot 49$ \\
$-3 \cdot 1$ & $-1 \cdot 15$ & $-4 \cdot 0$ & $-0 \cdot 24$ \\
$-3 \cdot 2$ & $-0 \cdot 87$ & $-5 \cdot 0$ & -0.06 \\
$-3 \cdot 3$ & -0.72 & $-6 \cdot 0$ & -0.01 \\
$-3 \cdot 4$ & -0.59 & $<-7 \cdot 0$ & 0.00 \\
\hline
\end{tabular}

straight line in Figure 2 if we remember the values of $Q$ and the probable values of $M_{\mathrm{H}}$. The simplest interpretation of this fact is that the upper luminosity limit $L_{1}$ in the exciting cluster or association is not much larger than $L_{1}$ and the function $G(L)$ is consequently reduced. This agrees qualitatively with the observation that earlytype galaxies appear to have a smaller upper-luminosity end.

TABLE 2

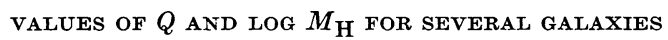

\begin{tabular}{l|c|c|c}
\hline \multicolumn{1}{c|}{ Object } & $\log M_{\mathrm{H}}$ & $Q$ & Source for $M_{\mathrm{H}}$ \\
\hline IC 1613 & $7 \cdot 69$ & $31 \cdot 3$ & \\
NGC 6822 & $8 \cdot 18$ & $31 \cdot 8$ \\
SMC & $8 \cdot 60$ & $31 \cdot 8$ \\
LMC & $8 \cdot 78$ & $32 \cdot 0$ \\
M51 & $8 \cdot 92$ & $33 \cdot 2$ & \\
M33 & $9 \cdot 00$ & $32 \cdot 2$ & Heidmann (1961) \\
Galaxy & $9 \cdot 18$ & $32 \cdot 4$ & Westerhout (1958) \\
NGC 300 & $9 \cdot 30$ & $32 \cdot 5$ & de Vaucouleurs and Page (1962) \\
M101 & $9 \cdot 47$ & $33 \cdot 3$ & W Heidmann (1961) \\
M31 & $9 \cdot 65$ & $31 \cdot 3$ & \} H
\end{tabular}

\section{References}

HeIDMAN, J. (1961).-B.A.N. 15: 314-18.

SANDAGE, A. R. (1957).-Ap. J. $125: 422-44$.

Schмidт, M. (1962).- "Symposium on Stellar Evolution." pp. 67-75. [La Plata Observatory 1960.] (Nat. Univ. La Plata Astron. Obs.)

Sersic, J. L. (1960).—Zs. f. Ap. 50 : 168-77.

de VAucouleurs, G., and PAGe, J. (1962).-Ap. J. $136: 107-18$.

WESTERHOUT, G. (1958).-B.A.N. 14: 215-60. 